\title{
Obstructive jaundice secondary to fungal infection: a rare case report
}

\author{
Weizhe Zhong ${ }^{1,2 \#}$, Ping Wang ${ }^{1,2 \#}$, Chuanyong Zhang ${ }^{1,2 \#}$, Zhuqing Rao $^{3}$, Xiaowei Wang ${ }^{1,2}$, Xiongxiong Pan $^{3}$, \\ Haoming Zhou ${ }^{1,2}$, Xuehao Wang ${ }^{1,2}$ \\ ${ }^{1}$ Hepatobiliary/Liver Transplantation Center, The First Affiliated Hospital with Nanjing Medical University, Nanjing, China; ${ }^{2}$ Research Unit of \\ Liver Transplantation and Transplant Immunology, Chinese Academy of Medical Sciences, Nanjing, China; ${ }^{3}$ Department of Anesthesiology, The \\ First Affiliated Hospital with Nanjing Medical University, Nanjing, China \\ \#These authors contributed equally to this work. \\ Correspondence to: Xuehao Wang; Haoming Zhou. Hepatobiliary/Liver Transplantation Center, The First Affiliated Hospital with Nanjing Medical \\ University; Research Unit of Liver Transplantation and Transplant Immunology, Chinese Academy of Medical Sciences, 300\# Guangzhou Road, \\ Nanjing, China. Email: wangxh@njmu.edu.cn; hmzhou@njmu.edu.cn.
}

\begin{abstract}
Obstructive jaundice is characterized by an obstruction of the intrahepatic or extrahepatic biliary system, and the most common causes include pancreatic and duodenal periampullary cancer. There have been some cases reporting obstructive jaundice caused by infection. Deep tissue infection usually develops in the individuals who are immunologically compromised or chronically ill, while a few cases reported in the immunocompetent patients. Those cases were diagnosed by fungal culture or percutaneous biopsy. Here, we presented an interesting case of obstructive jaundice secondary to fungal infection confirmed by postoperative pathological examination. A 79 years old man complaint about upper abdominal discomfort, darkened urine, and skin itch, with a history of esophageal cancer operation 5 years ago. The serology for hepatitis virus and human immunodeficiency virus (HIV) was negative. Imaging examinations showed a nodular located at distal common bile duct. As evidenced by increased level of cancer antigen 19-9 (CA19-9), the patient was highly suspected to be malignant obstructive jaundice. Thus, pylorus preserving pancreaticoduodenectomy (PPPD) was conducted. To our surprise, the ultimate diagnosis was fungal infection at the site of duodenum ampulla by the postoperative pathological examination, with no evidence of malignance. Anti-infective therapy was conducted subsequently, combined by fluconazole, sulperazone and tinidazole. Three weeks later, the patient was generally in good condition and discharged from hospital. During the 2-year follow-up, no fungal infection or tumor recurrence was observed. This case reminded us that fungal infection could be the cause of obstructive jaundice in an elderly person.
\end{abstract}

Keywords: Case report; obstructive jaundice; fungal infection; pylorus preserving pancreaticoduodenectomy; malignant tumor

Submitted Feb 25, 2020. Accepted for publication Jul 31, 2020.

doi: 10.21037/atm-20-1937

View this article at: http://dx.doi.org/10.21037/atm-20-1937

\section{Introduction}

Although the common causes of obstructive jaundice are choledocholithiasis and pancreatic cancer in the head of the pancreas, there have been some cases reporting obstructive jaundice caused by infection (1-3). Deep tissue infection usually develops in the individuals who are immunologically compromised or chronically ill, while a few cases reported in the immunocompetent patients. Those patients were diagnosed preoperatively, confirmed by fungal culture or percutaneous biopsy. Indeed, our patient was not suspected with fungal infection initially, as he had no respiratory or urinary infection in the recent 2 years, and no typical symptoms of infection such as fever and chills on his admission. Both laboratory and imagological 
Page 2 of 4

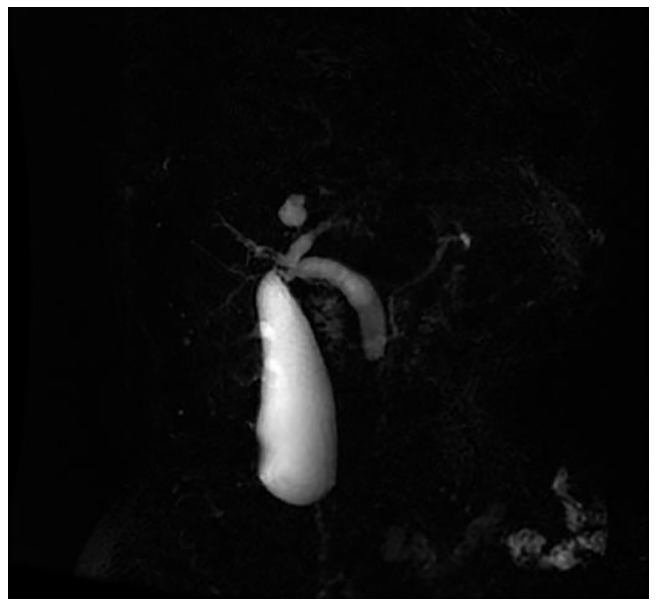

Figure 1 MRCP showed gall bladder enlargement and markedly dilated intra- and extrahepatic common bile duct.

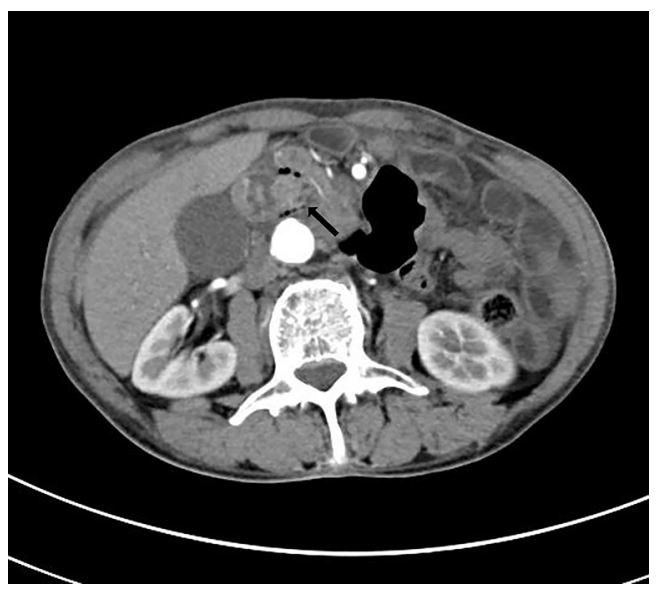

Figure 2 CT of the abdomen revealed a nodular of $1 \mathrm{~cm}$ in diameter (black arrowed) located at distal common bile duct with typical arterial enhancement.

findings indicated that he developed a malignant tumor. In such situation, we performed the pylorus preserving pancreaticoduodenectomy (PPPD) and found the fugus at the site of duodenum ampulla and distal common bile duct present article by postoperative histopathological examination. It was quite difficult to make correct diagnosis without a surgery biopsy. Summary, we reported fungal infection manifested as malignant obstructive jaundice in an elderly person.

We present the following article in accordance with the CARE reporting checklist (available at http://dx.doi.
Zhong et al. Obstructive jaundice secondary to fungal infection

org/10.21037/atm-20-1937).

\section{Case presentation}

A 79-year-old male complained of upper abdominal discomfort, darkened urine, itching, yellow discoloration of the eyes, and a $5 \mathrm{~kg}$ weight loss in one month. The patient had a history of radical resection for esophageal carcinoma at 5 years ago, without given chemotherapeutics. He had no respiratory or urinary infection in the recent 2 years.

On admission, he denied fever and chills, and no signs of peritoneal irritation were found in this patient. His total bilirubin was $2.44 \mathrm{mg} / \mathrm{dL}$ (normal, $0.3-1.1$ ), with a direct bilirubin of $1.6 \mathrm{mg} / \mathrm{dL}$ (normal, <0.4), aspartate amino transferase (AST), 329.5 U/L (normal, 15.0-40.0); alanine aminotransferase (ALT), 189.1 U/L (normal, 9.0-50.0), and g-glutamyl transpeptidase, 85.9 IU/L (normal, 10.0-60.0). The serology for hepatitis virus and HIV was negative. Increased serum level of CA19-9, 358 U/L (normal, <39) was detected, while serum level of carcinoembryonic antigen (CEA) was normal. Magnetic resonance cholangiopancreatography (MRCP) (Figure 1) showed gall bladder enlargement and markedly dilated intra-and extrahepatic common bile duct. A nodular of 1 $\mathrm{cm}$ in diameter located at distal common bile duct with typical arterial enhancement was revealed by computed tomography (CT) of the abdomen (Figure 2).

As highly suspected to be malignant obstructive jaundice, he received PPPD and the specimens was subject to histopathological examination subsequently. As shown in Figure 3, clusters of bluish-stained fungal colonies, spores and hyphae with acute inflammation were found at the site of duodenum ampulla and distal common bile duct (Figure 3). Fungal infection of ampulla of Vater was ultimately diagnosed, but no evidence of malignance was found by post-operative. Combination therapy of fluconazole ( $400 \mathrm{mg} / \mathrm{d}$, intravenous, 3 weeks), sulperazone $(6 \mathrm{~g} / \mathrm{d}$, intravenous, 1 week $)$ and tinidazole $(2 \mathrm{~g} / \mathrm{d}$, oral, 1 week) was given to this patient. He recovered well and discharged from hospital 3 weeks later. No fungal infection or tumor recurrence was observed during the 2-year follow-up.

We reported an interesting case that fungal infection manifested as malignant obstructive jaundice in an elderly person.

All procedures performed in studies involving human participants were in accordance with the ethical standards of 


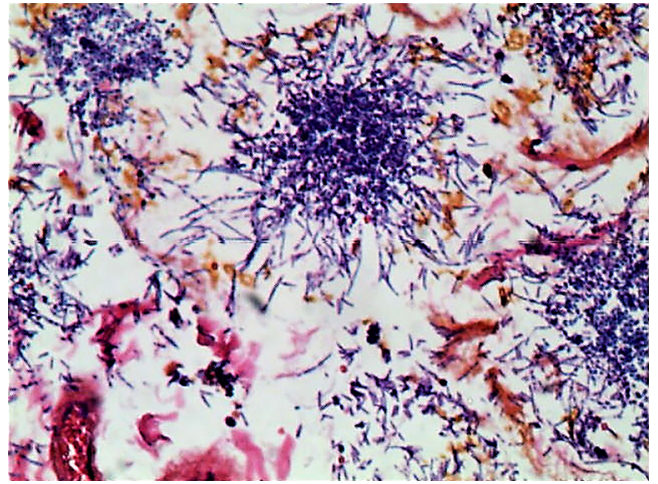

Figure 3 Post-operative histopathological examination (Gram stain, magnification $\times 100$ ) showed clusters of bluish-stained fungal colonies, spores, and hyphae with acute inflammation at the site of duodenum ampulla and distal common bile duct.

the institutional and national research committees, and with the Helsinki Declaration (as revised in 2013). Informed consent was obtained from the patient for the publication of their information and imaging.

\section{Discussion}

In the present article, we reported an interesting case that fungal infection manifested as malignant obstructive jaundice in an elderly person, who had a history of esophageal carcinoma. Deep fungal infection usually develops in immunocompromised hosts, such as patients infected with HIV, or treated with long term broadspectrum antibiotics or chemotherapeutics. No such risk factors were found in this present case, and this patient had no respiratory or urinary infection in the recent 2 years. Indeed, immunology is close to cancer. The decline of immunology function in the elderly could contribute to his past esophageal carcinoma development. A major limitation of our study is hard to define this 79-year-old male as immunocompetent or immunocompromised.

A radical surgery for esophageal carcinoma was performed on the patient 5 years ago. Recently, it was reported that unilateral endogenous fungal endophthalmitis occurred 2 months after esophageal cancer surgery (4), which indicated a possible relationship between the digestive tract surgery and the fungal infection. Impaired emptying of the gastric remnant following gastric resection and vagotomy could be responsible for the development of gastric mycosis (5). However, the underlying mechanism remains to be further studied.
Obstructive jaundice is characterized by an obstruction of the intrahepatic or extrahepatic biliary system. Most common causes include pancreatic and duodenal periampullary cancer. In addition, choledocholithiasis, stricture of the common bile duct, biliary atresia, pancreatitis, pancreatic pseudocyst, and parasite infection can lead to obstructive jaundice as well. In some cases, the differential diagnosis of inflammation and malignant tumor is difficult even under intraoperative biopsy. For example, tuberculosis of portal masses (6) had been reported to compress the common bile duct, performing like the malignance. The accurate diagnosis relies on the postoperative histopathological examination of whole-mount serial sections. The present case was preoperatively highly suspected with malignant obstructive jaundice, as supported by a lesion in distal common bile duct with typical arterial enhancement revealed by the CT scan, high levels of serum CA19-9, and history of esophageal carcinoma.

In conclusion, we have reported an extremely interesting and rare case of obstructive jaundice caused by fungal infection of ampulla of Vater. Fungal infection, the unusual cause of obstructive jaundice, needs to be considered for differential diagnosis of malignant tumor, which is essential to make proper treatments.

\section{Acknowledgments}

Funding: This work was supported by grants from National Nature Science Foundation of China (81530048, 81521004, 81470901, 81870448, 31930020, 81600450, 81901628), the National Science Foundation of Jiangsu Province (BK20191490), CAMS Innovation Fund for Medical Sciences (No. 2019-I2M-5-035, 2017PT32008, 2018PT31043, 2019PT320015), Six Talent Peaks Project in Jiangsu Province (No. 2018-WSN-011), Jiangsu Science and Technology Association Young Science and Technology Talents Lifting Project (No. DG000D4007) and A Project Funded by the PAPD.

\section{Footnote}

Reporting Checklist: The authors have completed the CARE reporting checklist. Available at http://dx.doi.org/10.21037/ atm-20-1937

Conflicts of Interest: All authors have completed the ICMJE uniform disclosure form. The authors have no conflicts of interest to declare (available at http://dx.doi.org/10.21037/ 


\section{Page 4 of 4}

atm-20-1937). XW serves as an unpaid editorial board member of Annals of Translational Medicine from Aug 2019 to Jul 2024. The other authors have no conflicts of interest to declare.

Ethical Statement: The authors are accountable for all aspects of the work in ensuring that questions related to the accuracy or integrity of any part of the work are appropriately investigated and resolved. All procedures performed in studies involving human participants were in accordance with the ethical standards of the institutional and national research committees, and with the Helsinki Declaration (as revised in 2013). Informed consent was obtained from the patient for the publication of their information and imaging.

Open Access Statement: This is an Open Access article distributed in accordance with the Creative Commons Attribution-NonCommercial-NoDerivs 4.0 International License (CC BY-NC-ND 4.0), which permits the noncommercial replication and distribution of the article with the strict proviso that no changes or edits are made and the original work is properly cited (including links to both the

\section{Zhong et al. Obstructive jaundice secondary to fungal infection}

formal publication through the relevant DOI and the license). See: https://creativecommons.org/licenses/by-nc-nd/4.0/.

\section{References}

1. Cazzo E, Ferrer JA, Chaim EA. Obstructive jaundice secondary to paracoccidioidomycosis. Trop Gastroenterol 2015;36:46-7.

2. Cai X, Liu K, Liang $Y$, et al. Isolated biliary cryptococcosis manifesting as obstructive jaundice in an immunocompetent adult. Int J Med Sci 2012;9:200-6.

3. Nara S, Sano T, Ojima H, et al. Liver cryptococcosis manifesting as obstructive jaundice in a young immunocompetent man: report of a case. Surg Today 2008;38:271-4.

4. Yi QY, Li WD, Chen LS, et al. Unilateral endogenous fungal endophthalmitis after esophageal cancer surgery: a case report. BMC Infect Dis 2018;18:671.

5. Rehnberg O, Faxen A, Haglund U, et al. Gastric mycosis following gastric resection and vagotomy. Ann Surg 1982;196:21-5.

6. Niemiec SM, Vinetz JM, Sicklick JK. Porta Hepatis Mass. JAMA Surg 2016;151:187-8.
Cite this article as: Zhong W, Wang $\mathrm{P}$, Zhang C, Rao Z, Wang X, Pan X, Zhou H, Wang X. Obstructive jaundice secondary to fungal infection: a rare case report. Ann Transl Med 2020;8(19):1250. doi: 10.21037/atm-20-1937 